\title{
Assessing the Real-Time Mental Health Challenges of COVID-19 in Individuals With Serious Mental IInesses: Protocol for a Quantitative Study
}

Raeanne Cristine Moore ${ }^{1}$, PhD; Colin Andrew Depp ${ }^{1,2}, \mathrm{PhD}$; Philip D Harvey ${ }^{3,4}, \mathrm{PhD}$; Amy E Pinkham ${ }^{5,6}, \mathrm{PhD}$

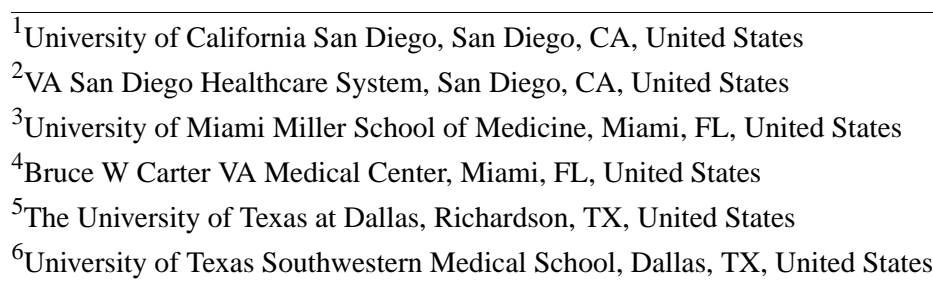

Corresponding Author:

Raeanne Cristine Moore, $\mathrm{PhD}$

University of California San Diego

220 Dickinson St, Ste B (8231)

San Diego, CA, 92103-8231

United States

Phone: 16195435378

Email: r6moore@ucsd.edu

\section{Abstract}

Background: The outbreak of coronavirus disease 2019 (COVID-19) has caused significant stress and mental health problems among the general public. However, persons at greatest risk for poor mental health outcomes, such as people with serious mental illness, have been largely overlooked.

Objective: This paper presents the protocol for a study that aims to examine the mental health impact of COVID-19 and social distancing behaviors in people with serious mental illness and the behaviors undertaken to prevent COVID-19 infection in this group.

Methods: Participants will include individuals with serious mental illness (eg, schizophrenia, bipolar disorder) and nonpsychiatric control participants who are currently participating in or have previously participated in several ongoing parent observational studies. Data will be collected from April 2020 through August 2020. Participants will complete phone interviews at 2 time points to assess their current emotional functioning and discuss the measures they have taken to prevent COVID-19 infection. Baseline (pre-COVID-19) mental health, sampled by ecological momentary assessment over an extended period, will be compared with current mental health, also sampled by ecological momentary assessment over an extended period. Demographic, cognitive, and psychosocial factors at baseline will be used to examine risk and resilience to current mental health and coping.

Results: The inclusion of participants for the first round of telephone assessments started on April 3, 2020 and will be completed by May 31, 2020. As of April 30, 2020, 101 individuals had completed these first-round assessments. The second round of telephone assessments will likely occur between June 1, 2020, and August 31, 2020. Study results will be published in peer-reviewed scientific journals.

Conclusions: Our findings will have broad implications for understanding the psychological consequences of COVID-19 among vulnerable persons with serious mental illness and will provide the opportunity to identify targets to reduce negative outcomes in the future. We also hope our efforts will provide a roadmap and resources for other researchers who would like to implement a similar approach.

International Registered Report Identifier (IRRID)： DERR1-10.2196/19203

(JMIR Res Protoc 2020;9(5):e19203) doi: 10.2196/19203

\section{KEYWORDS}

mental disorders; technology; telemedicine; pandemic; psychology; stress; social distancing; coping; COVID-19; public health 


\section{Introduction}

Coronavirus disease 2019 (COVID-19) has created a global pandemic and disrupted our society and daily lives. Americans have been forced to separate from their workplaces and their friends, engage in previously foreign behaviors including "social distancing" and "sheltering in place," and unemployment rates have jumped to unprecedented highs. The media have mainly focused on the psychological effects of COVID-19 in the general public, largely overlooking its impact on the most vulnerable groups in our society. The aim of this paper is to present the protocol for a study that will examine the effect of the pandemic on people with serious mental illness (SMI).

A recently published review in Lancet Psychiatry outlined the heightened risk of COVID-19 transmission among people with mental health disorders [1]. In addition to increased risk for infection, people with mental health disorders, and particularly those with SMI, could experience greater susceptibility to emotional responses to the pandemic, such as fear, anxiety, stress, depression, as well as risk of relapse or worsening of positive (eg, paranoia, hallucinations) and negative (eg, anhedonia, apathy) psychotic symptoms. This could be due to several reasons, including higher vulnerability to stress compared to the general population [2,3], reduced access to resources to permit ongoing mental health treatment and services [4,5], greater job or food insecurity [6,7], and additional restrictions in existing congregated situations such as group homes [8]. Social isolation or distancing may be less discrepant from daily living in some people with SMI than the general population. Moreover, some people with SMI may be less engaged in social networks and standard news media. Thus, the hypothesis of reduced subjective stress compared to the population in general needs to be considered.

On the other hand, health messages and awareness of the crisis are quite likely to not be well disseminated to people with SMI, creating a public health risk for individuals with SMI and others with whom they may have contact. An additional issue is challenges in the ability to understand and comply with complex directives and precautionary measures. People with SMI represent about $2 \%-3 \%$ of the population, and COVID-19 may result in collective increases in symptom severity, which, in turn, could result in expansive increases in mortality, emergency care utilization, and distress. Thus, it is critical to understand the influences of the COVID-19 pandemic on people with SMI. Further, our previous research has suggested that people with SMI are particularly challenged in self-assessments of both their emotional states and of their ability to engage in productive behaviors targeting their everyday functioning and self-management $[9,10]$.

Our research team has two ongoing studies centered around Strategy 3.1 of the National Institute of Mental Health's (NIMH) strategic plan to "identify and validate new targets for treatment development that underlie disease mechanisms" [11]. Both studies are multisite collaborations between the University of Texas at Dallas, University of California San Diego, and the University of Miami. Study 1 (principal investigator [PI]: author AEP, R01MH112620) assesses the construct of introspective accuracy, or the ability to correctly judge one's own skills and abilities. The goals of this study are (1) to learn how impaired introspective accuracy in individuals with serious mental illness contributes to difficulties in real-world functioning, (2) to understand how introspective accuracy differs from other types of self-awareness, and (3) to discover how clinical symptoms affect the amount and direction of introspective accuracy impairments among outpatients with serious mental illness. To date, 189 participants aged 18-60 years have completed the study protocol (101 with schizophrenia or schizoaffective disorder, 72 with bipolar disorder, 16 controls; see Table 1 for baseline demographic and clinical characteristics of the sample), which includes an in-person, lab-based assessment followed by 30 days of at-home symptom tracking and cognitive testing via smartphone-based ecological momentary assessment (EMA). The goals of study 2 (PI: author CAD, R01MH116902) are to understand, over a 1-year period, how cognitive biases in the ways that outpatients with psychotic disorders (eg, schizophrenia, bipolar disorder with psychosis) perceive other people impact suicidal ideation and behavior. Ninety-seven participants aged 18-65 years have completed baseline assessments (38 with schizophrenia, 41 with schizoaffective disorder, 16 with bipolar disorder with psychosis, 2 with major depressive disorder and psychosis; see Table 2 for baseline demographic and clinical characteristics of the sample). Similar to study 1, the baseline assessments for study 2 include an in-person, lab-based assessment followed by 10 days of in-the-moment reports of symptoms and performance-based social cognition assessments via smartphone-based EMA. 
Table 1. Participant demographic and clinical characteristics from parent study 1.

\begin{tabular}{|c|c|c|}
\hline Characteristic & Patients $(n=196)$ & Controls $(n=16)$ \\
\hline Sex (male), n (\%) & $88(45)$ & $11(69)$ \\
\hline \multicolumn{3}{|l|}{ Race, $\mathbf{n}(\%)$} \\
\hline Caucasian & $79(40)$ & $10(63)$ \\
\hline African American & $82(42)$ & $4(25)$ \\
\hline Native American & $3(2)$ & $0(0)$ \\
\hline Asian & $6(3)$ & $1(6)$ \\
\hline Native Hawaiian/Pacific Islander & $2(1)$ & 0() \\
\hline Other & $24(12)$ & $1(6)$ \\
\hline \multicolumn{3}{|l|}{ Ethnicity, n (\%) } \\
\hline Hispanic & $51(26)$ & $3(19)$ \\
\hline Non-Hispanic & $145(74)$ & $13(81)$ \\
\hline \multicolumn{3}{|l|}{ Diagnosis, $\mathrm{n}(\%)$} \\
\hline Schizophrenia & $60(31)$ & $\mathrm{N} / \mathrm{A}^{\mathrm{a}}$ \\
\hline Schizoaffective disorder & $52(27)$ & N/A \\
\hline Bipolar disorder (with psychotic features) & $45(23)$ & N/A \\
\hline Bipolar disorder (without psychotic features) & $38(19)$ & N/A \\
\hline \multicolumn{3}{|l|}{ Employment status ${ }^{\mathbf{b}}, \mathbf{n}(\%)$} \\
\hline Employed, full time & $22(11)$ & $13(81)$ \\
\hline Employed, part time & $25(13)$ & $1(6)$ \\
\hline Unemployed & $29(15)$ & $1(6)$ \\
\hline Stay-at-home parent & $2(1)$ & $0(0)$ \\
\hline Part-time student & $5(3)$ & $0(0)$ \\
\hline Full-time student & $6(3)$ & $2(13)$ \\
\hline Receiving disability & $96(49)$ & $0(0)$ \\
\hline Receiving disability, part-time work & $13(7)$ & $0(0)$ \\
\hline Retired & $6(3)$ & $0(0)$ \\
\hline \multicolumn{3}{|l|}{ Residential status $^{\mathbf{c}}, \mathbf{n}(\%)$} \\
\hline Independent, financially responsible & $136(69)$ & $16(100)$ \\
\hline Independent, not financially responsible & $38(19)$ & $0(0)$ \\
\hline Residential facility, unsupervised & $8(4)$ & $0(0)$ \\
\hline Residential facility, supervised & $13(7)$ & $0(0)$ \\
\hline Age (years), mean (SD) & $41.30(10.97)$ & $35.56(9.06)$ \\
\hline Education (years), mean (SD) & $13.30(2.57)$ & $15.13(1.09)$ \\
\hline Maternal education (years) ${ }^{\mathrm{d}}$, mean (SD) & $13.11(3.57)$ & $13.75(3.97)$ \\
\hline Paternal education (years) ${ }^{\mathrm{e}}$, mean (SD) & $13.59(3.74)$ & $14.69(2.56)$ \\
\hline \multicolumn{3}{|l|}{ Positive and Negative Syndrome Scale, mean (SD) } \\
\hline Positive total & $15.64(5.08)$ & N/A \\
\hline Negative total & $12.29(3.91)$ & N/A \\
\hline General total & $30.14(7.03)$ & N/A \\
\hline Montgomery-Asberg Depression Rating Scale total, mean (SD) & $10.70(10.65)$ & N/A \\
\hline Young Mania Rating Scale total, mean (SD) & $1.89(4.35)$ & N/A \\
\hline
\end{tabular}


${ }^{\mathrm{a}} \mathrm{N} / \mathrm{A}$ : not applicable.

${ }^{\mathrm{b}}$ Categories were not mutually exclusive.

${ }^{\mathrm{c}}$ Missing for 1 patient.

${ }^{\mathrm{d}}$ Missing for 29 patients.

${ }^{\mathrm{e}}$ Missing for 55 patients and 3 controls.

We also have one study that responds to NIMH Strategic Aim 2.2 [12] to develop novel behavioral assessments to evaluate domains relevant to mental illness. This is a single-site study at UCSD (PI: author RCM, R21MH116104) with the goals of understanding the real-time effects of mood on real-world cognitive performance and discovering how real-world cognition relates to real-time daily functioning among individuals with bipolar disorder. Sixty-six participants aged 18-65 years have completed this study (36 with bipolar disorder I, 10 with bipolar disorder II, 20 controls; see Table 3 for baseline demographic and clinical characteristics of the sample), which included a baseline assessment followed by 14 days of smartphone-based EMA and mobile cognitive testing (administered 3 times per day for a total possibility of 42 EMAs per participant).

For the present study, we will follow up with these previously enrolled research participants to assess their current mental health and psychosocial functioning with the exact same questions that were utilized during their previous participation. This study design will allow us to directly compare participants' prepandemic mental health functioning, based on dense

sampling of their momentary responses regarding symptoms, functioning, and self-evaluations with mental health functioning during the acute phase of the COVID-19 pandemic. We will also be positioned to examine demographic, cognitive, and psychosocial factors that may be predictive of better and worse mental health outcomes in this unprecedented time. Therefore, the aims of this study are to learn about (1) the mental health impact of COVID-19 and social distancing behaviors among at-risk populations and (2) prevention behaviors taken to reduce the risk of COVID-19 infection among persons with SMI. In so doing, we will use a comprehensive and detailed set of previously collected EMA data (up to 90 observations per patient collected over a 30-day sampling period) and ask those same questions again in two telephone reassessments: the first round of telephone assessments will occur between April 3, 2020, and May 31, 2020; the second round will occur 1 month after reopening. Although this will differ by state, we anticipate a date between June 1, 2020, and August 31, 2020. Our results should provide vital information regarding the overall level of awareness individuals with SMI have regarding the health risks of COVID-19 and how it is currently impacting their daily lives. 
Table 2. Participant demographic and clinical characteristics from parent study 2 (note: this study includes only individuals with a diagnoses of mental illness).

\begin{tabular}{lll}
\hline Characteristic & Suicidal ideation $(\mathrm{n}=48)$ & No suicidal ideation $(\mathrm{n}=49)$ \\
\hline Sex $($ male $), \mathrm{n}(\%)$ & $22(47)$ & $23(47)$
\end{tabular}

Race, $\mathbf{n}(\%)$

Caucasian

African American

Native American

Asian

Native Hawaiian/Pacific Islander

Other

Ethnicity, n (\%)

17 (36)

$0(0)$

$0(0)$

$3(6)$

$1(2)$

$0(0)$

$14(29)$

$2(4)$

\section{Hispanic}

$16(33)$

Non-Hispanic

$32(67)$

$42(86)$

Diagnosis, $n(\%)$

Schizophrenia

14 (29)

Schizoaffective disorder

$24(50)$

Bipolar disorder (with psychotic features)

8 (18)

Major depressive disorder (without psychotic features)

$1(2)$

$1(2)$

Employment status ${ }^{\mathrm{a}}$, n (\%)

Employed, full time

Employed, part time

Unemployed

4 (9)

Part-time student

$1(2)$

$0(0)$

Full-time student

$1(2)$

$0(0)$

Receiving disability

Receiving disability, part-time work

Retired

$0(0)$

\section{Residential status $^{\mathbf{b}}, \mathbf{n}(\%)$}

Independent, financially responsible

Independent, not financially responsible

$10(22)$

Residential facility, unsupervised

Residential facility, supervised

Age (years) ${ }^{\mathrm{c}}$, mean (SD)

44.04 (12.20)

$44.6(11.02)$

Education (years) ${ }^{\mathrm{d}}$, mean (SD)

$12.40(2.84)$

$12.81(1.86)$

Maternal education (years) ${ }^{\mathrm{e}}$, mean (SD)

$11.74(4.13)$

$12.63(3.44)$

Paternal education (years) ${ }^{\mathrm{f}}$, mean (SD)

$13.83(3.52)$

$13.14(3.55)$

Montgomery-Asberg Depression Rating Scale total ${ }^{\mathrm{g}}$, mean (SD)

$21.95(11.2)$

$9.68(9.86)$

Young Mania Rating Scale total ${ }^{\mathrm{h}}$, mean (SD)

$2.30(3.85)$

$1.21(3.38)$

${ }^{\mathrm{a}}$ Not available (ie, data not entered prior to shelter-in-place orders and unavailable at this time) for 3 patients with suicide ideation and 5 patients without suicide ideation.

${ }^{b}$ Not available for 3 patients with suicide ideation and 5 patients without suicide ideation.

${ }^{\mathrm{c}}$ Not available for 3 patients with suicide ideation and 5 patients without suicide ideation. 
${ }^{\mathrm{d}}$ Not available for 2 patients with suicide ideation and 5 patients without suicide ideation.

${ }^{\mathrm{e}}$ Missing for 10 patients with suicide ideation and 11 patients without suicide ideation.

${ }^{\mathrm{f}}$ Missing for 14 patients with suicide ideation and 16 patients without suicide ideation.

${ }^{\mathrm{g}}$ Total not available for 4 patients with suicide ideation and 5 patients without suicide ideation.

${ }^{\mathrm{h}}$ Total not available for 2 patients with suicide ideation and 5 patients without suicide ideation. 
Table 3. Participant demographic and clinical characteristics for parent study 3.

\begin{tabular}{|c|c|c|}
\hline Characteristic & Bipolar disorder $(n=46)$ & Controls $(n=20)$ \\
\hline Sex (male), n (\%) & $16(35)$ & $6(30)$ \\
\hline \multicolumn{3}{|l|}{ Race, n (\%) } \\
\hline Caucasian & $26(57)$ & $8(40)$ \\
\hline African American & $4(9)$ & $2(10)$ \\
\hline Native Hawaiian/Pacific Islander & $3(7)$ & $1(5)$ \\
\hline Other & $11(24)$ & $4(20)$ \\
\hline \multicolumn{3}{|l|}{ Ethnicity $^{\mathbf{a}}, \mathbf{n}(\%)$} \\
\hline Hispanic & $8(18)$ & $2(10)$ \\
\hline \multicolumn{3}{|l|}{ Diagnosis, n (\%) } \\
\hline Bipolar disorder I & $14(30)$ & $\mathrm{N} / \mathrm{A}^{\mathrm{b}}$ \\
\hline Bipolar disorder II & $10(22)$ & N/A \\
\hline Bipolar disorder I (with psychotic features) & $22(48)$ & N/A \\
\hline \multicolumn{3}{|l|}{ Employment status, n (\%) } \\
\hline Employed, full time & $13(28)$ & $12(60)$ \\
\hline Employed, part time & $4(9)$ & $4(20)$ \\
\hline Unemployed & $6(13)$ & $0(0)$ \\
\hline Stay-at-home parent & $0(0)$ & $0(0)$ \\
\hline Full-time student & $1(2)$ & $1(5)$ \\
\hline Receiving disability, unemployed & $16(35)$ & $0(0)$ \\
\hline Receiving disability, part-time work & $5(11)$ & $1(5)$ \\
\hline Retired & $1(2)$ & $2(10)$ \\
\hline \multicolumn{3}{|l|}{ Residential status, n (\%) } \\
\hline Independent, financially responsible & $36(78)$ & $16(80)$ \\
\hline Independent, not financially responsible & $8(17)$ & $4(20)$ \\
\hline Residential facility, unsupervised & $1(2)$ & $0(0)$ \\
\hline Residential facility, supervised & $1(2)$ & $0(0)$ \\
\hline Age (years), mean (SD) & $42.72(11.42)$ & $41.03(14.56)$ \\
\hline Education (years), mean (SD) & $14.91(2.52)$ & $15.65(2.74)$ \\
\hline Maternal education (years) ${ }^{\mathrm{c}}$, mean (SD) & $14.20(3.93)$ & $12.83(3.62)$ \\
\hline Paternal education (years) ${ }^{\mathrm{d}}$, mean (SD) & $15.22(3.04)$ & $15.24(3.07)$ \\
\hline Montgomery-Asberg Depression Rating Scale total, mean (SD) & $11.20(8.50)$ & N/A \\
\hline Young Mania Rating Scale total, mean (SD) & $6.44(5.64)$ & N/A \\
\hline
\end{tabular}

${ }^{\mathrm{a}}$ Missing for 1 participant with bipolar disorder.

${ }^{\mathrm{b}} \mathrm{N} / \mathrm{A}$ : not applicable.

${ }^{\mathrm{c}}$ Missing for 1 participant with bipolar disorder and 2 controls.

${ }^{\mathrm{d}}$ Missing for 9 participants with bipolar disorder and 3 controls. 


\section{Methods}

\section{Design}

This study involves 2 telephone interviews during which participants will be readministered psychiatric symptom-related questions that they received during the parent study via EMA, with the major modification being that the questions for the present study will be administered via a telephone survey. These items are presented in Multimedia Appendix 1 as a combination of the three surveys (please note that each parent study had a slightly different EMA survey). Participants will also be asked new questions about how they are currently feeling, thinking about, and dealing with COVID-19. The survey will take approximately 30 minutes to complete.

\section{Study Population and Inclusion and Exclusion Criteria}

All participants who are currently or have previously participated in one of our ongoing parent studies $(\mathrm{N}=352$ participants to date; approximately $24 \%$ participants overlap between studies), and who consented to being contacted for future studies, will be called and invited to participate. In general, participants include adults between the ages of 18 and 65 years who have a diagnosis of schizophrenia, schizoaffective disorder, bipolar disorder (I or II), or major depression with psychotic features. All individuals are receiving only outpatient care and are free from neurological and/or neurodegenerative disorders. A small sample of psychiatrically healthy individuals is also included $(\mathrm{n}=35)$.

\section{Questionnaires}

The parent study EMA questionnaires were developed by authors CAD, PDH, RCM, and AEP. All three questionnaires include items about engagement in daily activities and social interactions (Where are you? Who are you with? What are you doing?), mood (in the moment or since the past alarm), symptoms (eg, "since the past alarm, how often have you heard voices"), and other behavioral indicators of health (eg, sleep, substance use).

The newly developed COVID-19 exposure and prevention behavior questionnaire includes 16 items on exposure and prevention behaviors (Multimedia Appendix 2). We will also be administering open-source scales to assess the psychological impacts of COVID-19, including the Center for Epidemiologic Studies Depression Scale [13], National Institutes of Health PROMIS (Patient-Reported Outcomes Measurement Information System) emotional distress-anxiety scale [14], Perceived Stress Scale [15], 3 items from the UCLA Loneliness Scale [16], modified to be specific to COVID-19, the 6-item Lifetime Orientation Test-Revised (LOT-R; measure of optimism) [17], Satisfaction with Life Scale [18], Duke Social Support Scale-Social Interaction Subscale (4 items) [19], and an 11-item brief coping scale [20]. The corresponding author can be contacted to request a complete packet of these measures.

\section{Consent}

This study was approved by each participating university's Institutional Review Board. Participants will provide verbal

consent on the phone and will be compensated for their participation.

\section{Data Analysis Plan}

The estimated sample size is 200. For aim 1, the primary outcome will be change in average mood ratings (sadness, relaxed, energized, happiness, anxious) from the previous EMA surveys to now (spring 2020, when shelter-in-place orders are effective), then again during the summer of 2020 (unknown if shelter-in-place orders will be effective or if people have returned to a "normal" life). Changes in these outcomes will be evaluated using a mixed-models repeated measures analysis of variance with restricted maximum likelihood estimation. Group membership (schizophrenia, schizoaffective disorder, bipolar disorder) and assessment point (baseline, follow-up) will be treated as fixed effects and participants will be treated as a random effect. The group-by-time interaction will be the fixed effect of interest. Secondary analyses will be conducted to evaluate (1) the predictors of change from baseline, with a focus on diagnosis and psychotic symptoms, examined with regression models, and (2) group differences at each time point and differences in change scores between controls $(n=35)$ and patient groups.

For aim 2, the primary outcome will be characterization of prevention behavior by group. For studies and participants where we have this information, we will also relate these data to assessments of insight, including clinical insight, self-monitoring ability collected during EMA, and the results of a comprehensive assessment of the ability to evaluate one's own performance on an array of neurocognitive, social cognitive, and functional measures. These analyses will be examined with correlational statistics, including regression models.

\section{Results}

The inclusion of participants for the first round of telephone assessments started on April 3, 2020 and will be completed by May 31, 2020. As of April 30, 2020, 101 individuals had completed these first-round assessments. The second round of telephone assessments will likely occur between June 1, 2020, and August 31, 2020. Study results will be published in peer-reviewed scientific journals in a timely fashion at completion of data collection. Data addressing non-COVID-19 topics from the sample collected to date are already being submitted for publication to scientific journals.

\section{Discussion}

\section{Principal Findings}

This study will shed light on the direct impact of the COVID-19 pandemic on the well-being of people with serious mental illness, a largely overlooked yet vulnerable population during this pandemic. Individuals with mental illness are often burdened not only by their illness but also by social isolation, under- or unemployment, lower socioeconomic status, cognitive impairments, and limited access care. Such individuals may therefore represent a particularly vulnerable and important group in whom we must strive to understand the effects of COVID-19. Findings from this study have the potential to characterize the 
degree of distress among persons with SMI during this pandemic and will also help to clarify whether individuals with SMI are able to protect themselves and others from infection. These findings can also help us identify risk and resiliency factors predictive of positive and negative outcomes to this high-stress situation, which could provide targets for early intervention in the (likely inevitable) event that another pandemic occurs and/or that social distancing measures are necessary in the future. Lastly, we hope this protocol paper will provide a roadmap and resources for other researchers who would like to implement a similar approach in their studies.

\section{Authors' Contributions}

All authors contributed extensively to the work presented in this paper. RCM designed the protocol and wrote the paper; CAD, PDH, and AEP wrote the paper. RCM, CAD, and AEP are the PIs on the parent grants of which this study is an extension of.

\section{Conflicts of Interest}

RCM is a cofounder and vice president of research of KeyWise AI, Inc. She has a research grant from Gilead Sciences. PDH has received consulting fees or travel reimbursements from Acadia Pharma, Alkermes, Bio Excel, Boehringer Ingelheim, Minerva Pharma, Otsuka Pharma, Regeneron Pharma, Roche Pharma, and Sunovion Pharma during the past year. He receives royalties from the Brief Assessment of Cognition in Schizophrenia. He is chief scientific officer of i-Function, Inc. He had a research grant from Takeda and the Stanley Medical Research Foundation. The remaining authors declare that they have no conflicts of interest pertinent to this study.

\section{Multimedia Appendix 1}

Psychiatric symptom EMA questions from parent studies (combined), adapted for a telephone follow-up assessment. Includes skip-logic.

[DOCX File, $51 \mathrm{~KB}-$ Multimedia Appendix 1]

\section{Multimedia Appendix 2}

COVID-19 exposure and prevention behavior questionnaire.

[DOCX File, 17 KB-Multimedia Appendix 2]

\section{References}

1. Yao H, Chen J, Xu Y. Patients with mental health disorders in the COVID-19 epidemic. The Lancet Psychiatry 2020 Apr;7(4):e21. [doi: 10.1016/S2215-0366(20)30090-0] [Medline: 32199510]

2. Norman RM, Malla AK. Stressful life events and schizophrenia. I: A review of the research. Br J Psychiatry 1993 Feb;162:161-166. [doi: 10.1192/bjp.162.2.161] [Medline: $\underline{8435685]}$

3. Phillips LJ, Francey SM, Edwards J, McMurray N. Stress and psychosis: towards the development of new models of investigation. Clin Psychol Rev 2007 Apr;27(3):307-317. [doi: 10.1016/j.cpr.2006.10.003] [Medline: 17169470]

4. Hall JP, LaPierre TA, Kurth NK. Medicaid managed care: issues for enrollees with serious mental illness. Am J Manag Care 2019 Sep;25(9):450-456 [FREE Full text] [Medline: $\underline{31518095]}$

5. Lawrence D, Kisely S. Inequalities in healthcare provision for people with severe mental illness. J Psychopharmacol 2010 Nov;24(4 Suppl):61-68 [FREE Full text] [doi: 10.1177/1359786810382058] [Medline: 20923921]

6. Cook JA. Employment barriers for persons with psychiatric disabilities: update of a report for the President's Commission. Psychiatr Serv 2006 Oct;57(10):1391-1405. [doi: 10.1176/ps.2006.57.10.1391] [Medline: 17035556]

7. Mangurian C, Sreshta N, Seligman H. Food insecurity among adults with severe mental illness. Psychiatr Serv 2013 Sep 01;64(9):931-932 [FREE Full text] [doi: 10.1176/appi.ps.201300022] [Medline: 24026843]

8. Fakhoury WKH, Murray A, Shepherd G, Priebe S. Research in supported housing. Soc Psychiatry Psychiatr Epidemiol 2002 Jul;37(7):301-315. [doi: 10.1007/s00127-002-0549-4] [Medline: 12111022]

9. Silberstein JM, Pinkham AE, Penn DL, Harvey PD. Self-assessment of social cognitive ability in schizophrenia: Association with social cognitive test performance, informant assessments of social cognitive ability, and everyday outcomes. Schizophr Res 2018 Sep;199:75-82 [FREE Full text] [doi: 10.1016/j.schres.2018.04.015] [Medline: 29673732]

10. Sabbag S, Twamley EW, Vella L, Heaton RK, Patterson TL, Harvey PD. Predictors of the accuracy of self assessment of everyday functioning in people with schizophrenia. Schizophr Res 2012 May;137(1-3):190-195 [FREE Full text] [doi: 10.1016/j.schres.2012.02.002] [Medline: 22386735]

11. National Institute of Mental Health. 2019. Priorities for Strategy 3.1 URL: https://www.nimh.nih.gov/about/ strategic-planning-reports/strategic-research-priorities/srp-objective-3/priorities-for-strategy-31.shtml [accessed 2020-04-05]

12. National Institute of Mental Health. 2019. Priorities for Strategy 2.2 URL: https://www.nimh.nih.gov/about/ strategic-planning-reports/strategic-research-priorities/srp-objective-2/priorities-for-strategy-22.shtml [accessed 2020-04-05] 
13. Andresen EM, Malmgren JA, Carter WB, Patrick DL. Screening for depression in well older adults: evaluation of a short form of the CES-D (Center for Epidemiologic Studies Depression Scale). Am J Prev Med 1994;10(2):77-84. [Medline: 8037935]

14. Cook KF, Jensen SE, Schalet BD, Beaumont JL, Amtmann D, Czajkowski S, et al. PROMIS measures of pain, fatigue, negative affect, physical function, and social function demonstrated clinical validity across a range of chronic conditions. J Clin Epidemiol 2016 May;73:89-102 [FREE Full text] [doi: 10.1016/j.jclinepi.2015.08.038] [Medline: 26952842]

15. Cohen S, Kamarck T, Mermelstein R. A global measure of perceived stress. J Health Soc Behav 1983 Dec;24(4):385-396. [Medline: $\underline{6668417]}$

16. Russell DW. UCLA Loneliness Scale (Version 3): reliability, validity, and factor structure. J Pers Assess 1996 Feb;66(1):20-40. [doi: 10.1207/s15327752jpa6601_2] [Medline: 8576833]

17. Scheier MF, Carver CS, Bridges MW. Distinguishing optimism from neuroticism (and trait anxiety, self-mastery, and self-esteem): a reevaluation of the Life Orientation Test. J Pers Soc Psychol 1994 Dec;67(6):1063-1078. [doi: 10.1037//0022-3514.67.6.1063] [Medline: 7815302]

18. Diener E, Emmons RA, Larsen RJ, Griffin S. The Satisfaction With Life Scale. J Pers Assess 1985 Feb;49(1):71-75. [doi: 10.1207/s15327752jpa4901 13] [Medline: 16367493$]$

19. Blazer D, Hybels C, Hughes D. Duke social support index. Princeton, NJ: Educational Testing Service; 1990.

20. Carver CS. You want to measure coping but your protocol's too long: consider the brief COPE. Int J Behav Med 1997;4(1):92-100. [doi: 10.1207/s15327558ijbm0401 6] [Medline: 16250744$]$

\author{
Abbreviations \\ COVID-19: coronavirus disease 2019 \\ EMA: ecological momentary assessment \\ NIMH: National Institute of Mental Health \\ PI: principal investigator \\ SMI: serious mental illness
}

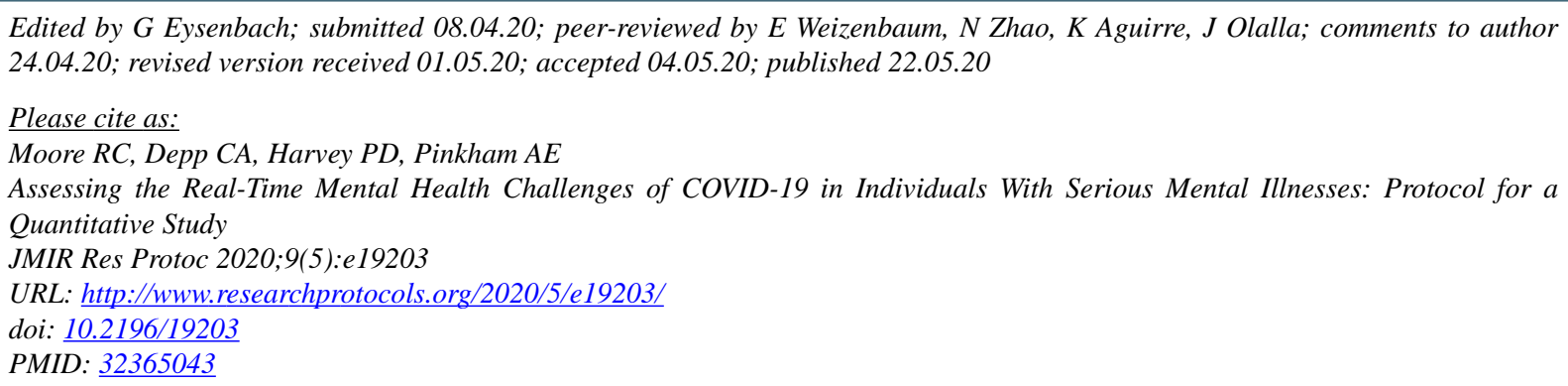

CRaeanne Cristine Moore, Colin Andrew Depp, Philip D Harvey, Amy E Pinkham. Originally published in JMIR Research Protocols (http://www.researchprotocols.org), 22.05.2020. This is an open-access article distributed under the terms of the Creative Commons Attribution License (https://creativecommons.org/licenses/by/4.0/), which permits unrestricted use, distribution, and reproduction in any medium, provided the original work, first published in JMIR Research Protocols, is properly cited. The complete bibliographic information, a link to the original publication on http://www.researchprotocols.org, as well as this copyright and license information must be included. 